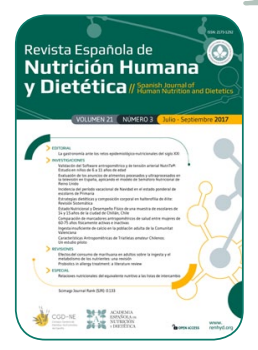

\title{
EDITORIAL
}

\section{La gastronomía ante los retos epidemiológico-nutricionales del siglo XXI}

\author{
Josep Bernabeu-Mestre ${ }^{a}$, María Eugenia Galiana Sánchez ${ }^{a}$, Eva María Trescastro López ${ }^{a, *}$

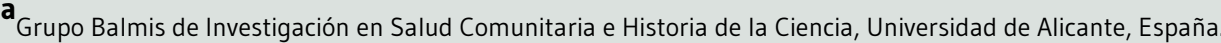 \\ *eva.trescastro@ua.es
}

Recibido el 20 de julio de 2017; aceptado el 15 de septiembre de 2017; publicado el 3 de octubre de 2017.

\section{CITA}

Bernabeu-Mestre J, Galiana Sánchez ME, Trescastro López EM. La gastronomía ante los retos epidemiológiconutricionales del siglo XXI. Rev Esp Nutr Hum Diet. 2017; 21(3): 209-12. doi: 10.14306/renhyd.21.3.438

La alimentación ha jugado un papel fundamental en el desarrollo de la humanidad, ha contribuido a su configuración social y cultural y se ha convertido en un factor clave para explicar las dinámicas demográficas y de salud ${ }^{1}$.

Sin embargo, a pesar de todas estas evidencias, en el siglo $X X I$, en materia de alimentación y nutrición, el ser humano tiene que seguir haciendo frente al hambre y la desnutrición -como expresión biológica del subdesarrollo y la desigualdad social ${ }^{2}$-, al mismo tiempo que debe afrontar el reto de una pandemia de obesidad que responde, por un lado, a la sobrealimentación y los hábitos alimentarios inadecuados, $y$, por otro, a lo que se conoce como la obesidad de la pobreza, aquella que convive con el hambre y la desnutrición y que comparte buena parte de sus factores determinantes ${ }^{3}$.
Para superar este doble reto, se debe garantizar a todas las personas una alimentación de calidad donde la gastronomía está llamada a jugar un papel fundamental.

Desde la sinergia que cabe establecer entre la nutrición y la evolución de la propia gastronomía y de la cocina, el saber gastronómico, entendido como una ciencia y un arte que nos suministra los conocimientos necesarios para la elección de los alimentos convenientes, y cómo proceder al condimento de los mismos y a su presentación en la mesa ${ }^{4}$, debe evolucionar en beneficio de la nutrición. Con la gastronomía podemos aprender a comer y a nutrirnos de forma adecuada, sin renunciar al objetivo de disfrutar comiendo. La cocina debe apostar por una gastronomía centrada en las materias primas de calidad y asegurar, así, el sabor de los alimentos y la salud de los consumidores ${ }^{5}$. 
Hoy asociamos la cocina con el hecho de hacer a los alimentos más apetitosos, pero en un principio eran cocinados para facilitar su digestión, para hacerlos comestibles. Como señala Almudena Villegas 6 "la gastronomía aparece cuando las necesidades primarias están satisfechas, cuando el ser humano elabora y recrea el alimento que ya no es una afán prioritario y cotidiano". En palabras de Josep María Pinto7, la gastronomía aparece en el momento en el que se introducen en el imprescindible acto de comer nuevos parámetros: el placer, la sociabilidad, la reflexión (y añadimos, nosotros, también la salud).

Como recuerda Rafael Ansón ${ }^{8}$, hoy más que nunca la gastronomía debe asumir su doble perfil, y adoptar su condición de concepto unitario, ya que no resulta posible disociar los aspectos que afectan a la salud de los componentes vinculados con el placer. Se trata, sostiene dicho autor, de pasar de una época donde lo único importante eran el placer y la satisfacción, a una sociología de la alimentación que implica no sólo acabar con el hambre y tratar de que todas las personas coman saludablemente, sino, también y de una forma muy especial, que cada vez más personas disfruten comiendo. Que el placer gastronómico no corresponda sólo a unos cuantos privilegiados, sino que se extienda a la mayoría de las poblaciones.

Ha de ser desde la confluencia entre nutrición y gastronomía, como se debe intentar alcanzar una alimentación de calidad, aquella que además de ser nutricionalmente adecuada, variada y saludable, sea rica, apetecible y adaptada a los gustos y necesidades de los consumidores. Se trata de incorporar la calidad gastronómica, y contemplar las características organolépticas de los alimentos (sabor, olor, color, textura, etc.), de las técnicas y métodos empleados en su preparación y cocinado, así como de la habilidad aplicada a las mismas, y de factores más relativos, variables o subjetivos, tales como los gustos individualizados, o los usos y las modas de la época, lugar o cultura ${ }^{9}$. No se come sólo por salud, también se come por placer, y, sobre todo, se come de acuerdo con unos hábitos alimentarios. Al programar una dieta, aunque sea correcta desde el punto de vista nutricional, si no se tiene en cuenta el placer y los hábitos, es decir la gastronomía, muy probablemente fracasará ${ }^{5}$.

Como recordaba el profesor Gregorio Varela Mosquera ${ }^{10}$, "un alimento si no se come no cumple su misión". Existen tres elementos que determinan el consumo o no consumo de un alimento: su palatabilidad, su digestibilidad y su metabolicidad, siendo la primera la llave para los otros dos. El reto de conseguir la palatabilidad se convierte, así, en el punto de encuentro entre gastronomía y nutrición, y en uno de los factores fundamentales en el desarrollo de la gastronomía. Los gastrónomos buscan conocer la influencia de los diferentes procesos culinarios en la palatabilidad de sus elaboraciones, a través de las informaciones que proporcionan las ciencias de la nutrición y los alimentos ${ }^{11}$.

Se trata de llevar a la práctica las enseñanzas del buen comer, logrando coordinar los aspectos nutricionales con los gastronómicos. Pero se trata también de recordar que la alimentación es un derecho básico y una responsabilidad colectiva que precisan de una cultura alimentaria basada en una gastronomía saludable ${ }^{8}$.

El reto está en formar ciudadanos gastronómicamente responsables. Comer mejor significa vivir mejor ('somos lo que comemos"), y aunque comer bien pueda resultar complejo, también debería resultar placentero. Promover y preservar la salud pasa por desarrollar y adquirir unos hábitos alimentarios adecuados, de ahí la importancia de integrar en el discurso de la nutrición humana y la dietética, la idea de que la gastronomía constituye uno de los pilares fundamentales de la cultura de la salud, y, por ello, los ciudadanos preservarán mejor su salud cuando mejor desarrollen sus hábitos alimentarios, incluyendo en los mismos la plena recuperación de la función social que implica el hecho de alimentarse ${ }^{8}$.

En el proceso de humanización de la conducta alimentaria, el comer se convirtió en un acto social, y en dicho proceso la gastronomía jugó un papel destacado. Como afirmaba el profesor Gregorio Varela Mosquera ${ }^{10}$, "El ser humano es el único animal que cocina sus alimentos, y además el único que los comparte. Sólo él produce una cocina gastronómica". Sin embargo, en los últimos tiempos, y a pesar de la actualidad y la importancia mediática que muestran tanto la alimentación como la gastronomía, parece que estamos revirtiendo los efectos de la revolución culinaria que convirtió el acto de comer en acto saludable y socializador.

Los alimentos precocinados se han convertido, de hecho, en un emblema de la cultura del calentar y servir, del plástico y del silencio, donde se ha instalado el consumidor de comida rápida. La comodidad de los alimentos preparados ha comportado, también, un cambio importante de valores y ha conllevado la generalización de productos procesados industrialmente concebidos para ser consumidos a toda prisa o bien delante del ordenador o de la televisión ${ }^{12}$. ¿Hasta qué punto corremos el peligro de retroceder en el efecto socializador que acompañó la primera gran revolución de la comida?, ¿estamos priorizando la comodidad y la rapidez frente al placer de comer o el objetivo de nutrirse de forma saludable?, ¿dónde queda el saber gastronómico bien entendido?

Para conseguir que la alimentación-nutrición y la alimentación-gastronomía cumplan con el papel que tienen que desempeñar en las sociedades actuales, parece indispensable 
que los conocimientos de alimentación-nutrición y la educación del gusto, es decir, la educación en materia de alimentación y gastronomía se incorporen, como algo absolutamente esencial y obligatorio, al sistema educativo ${ }^{13}$, incluida la formación gastronómica de los dietistas-nutricionistas ${ }^{14}$.

Como se subrayaba en la iniciativa aprobada por el Parlamento Europeo sobre El Patrimonio Gastronómico Europeo: Aspectos Culturales y Educativos, el 12 de marzo de $2014^{13}$, si se quieren evitar gastos extraordinarios y difícilmente asumibles, incluso en las sociedades más desarrolladas, para curar las enfermedades y las patologías derivadas de una mala alimentación, la gastronomía debe incorporarse a las aulas.

Para Rafael Ansón ${ }^{8}$, el objetivo principal de la educación y la cultura alimentaria del siglo XXI tiene que ser demostrar y convencer a todo el mundo, que es absolutamente compatible, además de obligatorio, comer saludable y gastronómicamente.

La dieta gastronómica ${ }^{15}$, entendida como aquella que integra el discurso de la nutrición humana y la dietética, al mismo tiempo que ofrece platos atractivos y apetitosos -indicando el modo de elaborarlos y en ocasiones modificando la forma tradicional de confeccionarlos-, se puede convertir en un instrumento válido para alcanzar las recomendaciones nutricionales de energía y nutrientes, pero sin olvidar que no se come únicamente para no enfermar y mantener la salud, sino también por placer y por unos hábitos alimentarios que son consecuencia de una historia sociocultural.

En este sentido, la cultura alimentaria mediterránea ${ }^{16}$ en consonancia con movimientos como el Slow Food ${ }^{17}$, nos aporta un patrimonio gastronómico que hace referencia al complejo entramado de prácticas y conocimientos, valores y creencias, técnicas y representaciones sobre qué, cuándo, cómo, con quién, y por qué se come lo que se come. Incluye los productos y las técnicas de producción o elaboración, y también usos y costumbres y formas de consumo ${ }^{18,19}$.

Nos enfrentamos al reto de conseguir una cocina y una forma de alimentarnos (la gastronomía) compatible con los descubrimientos más recientes de la nutrición. ¿Por qué no adoptamos y/o readaptamos la Dieta Mediterránea en los términos que cabría esperar? Muy probablemente porque elegir un estilo de vida saludable, como el que representa la Dieta Mediterránea, no depende únicamente de la voluntad (el querer), si no que entran en juego el saber (informar y educar) y el poder (la accesibilidad). Hacer compatibles las tres condiciones, exige información y educación, pero también políticas públicas que garanticen para todos los ciudadanos la accesibilidad a una gastronomía saludable.

\section{CONFLICTO DE INTERESES}

Los autores expresan que no hay conflictos de interés al redactar el manuscrito.

\section{$\longrightarrow$ REFERENCIAS}

(1) Bernabeu-Mestre J, Esplugues JX, Trescastro EM. Evolución histórica de las estrategias alimentarias y sus condicionantes [Internet]. Alicante: Universitat d'Alacant; 2014. Disponible en: http://rua.ua.es/dspace/handle/10045/45087

(2) Food and Agriculture Organization of the United Nations. FAO in the 21st century: ensuring food security in a changing world. Rome: FAO; 2011.

(3) Caballero B. The global epidemic of obesity: an overview. Epidemiol Rev. 2007; 29: 1-5.

(4) Esplugues JX, Galiana ME, Pascual M, Trescastro E, BernabeuMestre J. Nutrició i salut en els textos de gastronomia alacantina: I’obra de José Guardiola Ortiz (1872-1946). En: Pascual M, Esplugues JX, Galiana ME, Trescastro E, BernabeuMestre J, editores. Turisme, gastronomia, oci i salut als municipis valencians: una perspectiva històrica. San Vicent del Raspeig, España: Seminari d’Estudis sobre la Ciencia; 2012.

(5) Ansón R, Varela G. Gastronomía saludable. León, España: Everest; 2007.

(6) Villegas A. Saber del sabor: manual de cultura gastronómica. Córdoba, España: Almuzara; 2008.

(7) Pinto JM. Recorrido histórico. En: AAVVV Curso de Gastronómica y Ciencias de la Alimentación. Manual para estudiantes. Núcleo tercero. Las tecnologías Alimentarias y la Cocina. Creatividad. Gijón: Universidad Camilo José Cela / Cátedra Ferrán Adrià de Cultura Gastronómica y Ciencias de la Alimentación; 2005. p. 60-77.

(8) Ansón R. Visión Global de la Gastronomía en el siglo XXI Discurso Académico de D. RAFAEL ANSÓN OLIART, Presidente de la Real Academia de Gastronomía [Internet]. Real Academia de Gastronomía. 2014. Disponible en: http://www. realacademiadegastronomia.com/discurso.php?id=2

(9) Cruz J. Teoría elemental de la gastronomía. Barañáin (Navarra): EUNSA; 2002.

(10) Varela Mosquera, G. Prólogo. En: Llona Larrauri J. Ciencia, curiosidades y mitos gastronómicos. Bilbao: Editorial La Gran Enciclopedia Vasca; 1995. p. 12-13.

(11) Castells P. La Cocina del futuro: cocina, ciencia y salud. Barcelona: Tibidabo; 2016.

(12) Bengoa y Lecanda JM. Reflexiones: Las raíces de la alimentación vasca. En: Tras la ruta del hambre: nutrición y salud pública en el siglo XX. Alicante: Universidad de Alicante; 2005. (Publicaciones de la Universidad de Alicante).

(13) Parlamento Europeo. Patrimonio gastronómico europeo Resolución del Parlamento Europeo, de 12 de marzo de 2014, sobre el patrimonio gastronómico europeo: aspectos culturales y educativos (2013/2181(INI)) [Internet]. 2014. Disponible en: 
http://www.europarl.europa.eu/sides/getDoc.do?pubRef=-// EP//NONSGML+TA+P7-TA-2014-0211+0+DOC+PDF+VO//ES

(14) Trescastro EM, Bernabeu-Mestre J, Esplugues JX, Galiana ME, Tormo M. 0-076: Video recipes provide an ideal way of both promoting healthy eating and for recovering our culinary traditions. En: 17th International Congress of Dietetics (ICD): going to sustainable eating - Granada, 2016. Rev Esp Nutr Hum Diet. 2016; 20(suppl 1): 401-2.

(15) Luzón A. La dieta gourmet: método Luzón: cómo adelgazar sin renunciar al placer. Torrelodones, Madrid: El Sindicato del Gusto; 2013.

(16) Instituto Tomás Pascual Sanz, Universidad San Pablo CEU, Universidad Cardenal Herrera-CEU. ¿Es posible la dieta mediterránea en el siglo XXI? Madrid: Instituto Tomás Pascual
Sanz Universidad San Pablo CEU; Universidad Cardenal Herrera CEU; 2011.

(17) Slow Food. Bienvenidos a nuestro mundo: La guía [Internet]. Bra, Italia: Slow Food; 2008. Disponible en: http://www. slowfoodib.org/pdf/descargas/N\%C2\%BA1A-GUIA\%20 RAPIDA\%20Bienvenidos\%20a\%20Slow\%20Food.pdf

(18) Castells M. El Reconocimiento Internacional de la Dieta Mediterránea como Patrimonio Inmaterial: Oportunidades para el Turismo Gastronómico Balear. Boletín Gestión Cultural [Internet]. 2008;17. Disponible en: http://www. gestioncultural.org/boletin/2008/bgc17-MCastells.pdf

(19) Bernabeu-Mestre J, Galiana-Sánchez ME, Trescastro EM. EI paper de I'etnobotànica en la consecució d'una alimentació saludable. Randa. 2015; 75: 173-85. 